\title{
Synthesis and Characterization of Water Soluble Choline Labeled Cadmium Selenide/Zinc Selenide/Zinc Sulfide Luminescent Quantum Dots
}

\author{
Kristi L. Mock, ${ }^{\text {a }}$ L. M. Viranga Tillekeratne ${ }^{\mathrm{a}, \mathrm{b}, \mathrm{c}}$ and Jon R. Kirchhoff ${ }^{\mathrm{a}, \mathrm{b}, \mathrm{c}}$ \\ ${ }^{a}$ Department of Chemistry and Biochemistry, College of Natural Sciences and Mathematics \\ ${ }^{b}$ Department of Medicinal and Biological Chemistry, College of Pharmacy and Pharmaceutical \\ Science \\ ${ }^{\mathrm{c}}$ School of Green Chemistry and Engineering. University of Toledo, 2801 West Bancroft Street, \\ Toledo, $\mathrm{OH} 43606$
}

*Corresponding Author

Jon R. Kirchhoff

Department of Chemistry and Biochemistry

The University of Toledo

Toledo, $\mathrm{OH} 43606$

e-mail: jonkirchhoff@utoledo.edu

phone: (419) 530-1515

Fax: (419) 530-4033

\begin{abstract}
Water soluble choline-labeled $\mathrm{CdSe} / \mathrm{ZnSe} / \mathrm{ZnS}$ quantum dot (QD) bioconjugates were synthesized by attaching a thiolated choline analogue to the core-shell QD surface. Characterization was conducted by absorption and luminescence spectroscopy and scanning electron microscopy. QDs with diameters of 5-6 nm resulted and exhibited a luminescence maximum at $663 \mathrm{~nm}$ in aqueous solution.
\end{abstract}

Keywords. CdSe/ZnSe/ZnS quantum dots, neurotransmission, choline, Alzheimer's disease, choline uptake

Neurodegenerative diseases are debilitating progressive disorders with no known cure. For example, Alzheimer's disease (AD) is the sixth leading cause of death in the United States [1] affecting 5.4 million, mainly elderly Americans. [2] Although AD was first described in 1906, [3] the cause of neuronal breakdown is complicated and the subject of significant research. Indications of $\mathrm{AD}$ have been associated with accumulation of $\beta$-amyloid proteins in the region between neurons, [4] the buildup of insoluble twisted tau fibers in neurons, [5] and the breakdown of the myelin sheath surrounding neurons. [6] Degradation of various neurotransmitter systems, including selective degradation of cholinergic neurons, also has been observed with a decrease in brain concentrations of the neurotransmitter acetylcholine (ACh), its precursor (Ch, Figure 1a), and proteins that mediate their concentrations such as acetylcholinesterase, the high affinity choline transporter (CHT), and choline acetyl transferase.[7] 
Normal cholinergic function requires synthesis of ACh in the neuron from Ch imported by CHT. [8] Therefore, $\mathrm{Ch}$ transport is a critical process and studies have focused on understanding the structure and function of CHT through the synthesis of $\mathrm{Ch}$ analogues with cholinomimetic features. [9] Even so, structural information for Ch transporters is limited [10] and often inferred from other biogenic amine transport proteins. [9] Since $\mathrm{Ch}$ is both hydrophilic and a positively charged ion, passive diffusion across the cell membrane is unlikely. Studies have shown that recognition of $\mathrm{Ch}$ by CHT was dependent upon the presence of both the quaternary ammonium and hydroxyl groups among other subtle factors. [11] Such studies have relied extensively on radiochemical methods in the presence and absence of CHT inhibitors. [9] An alternate non-radiochemical approach was developed in our laboratory using capillary electrophoresis with electrochemical detection to measure small time-dependent changes in $\mathrm{Ch}$ concentrations in vitro and in vivo under conditions of neuronal degradation with attomole detection limits and $\mathrm{nL}$-sized samples. [12]

Quantum dot (QD) semiconductor nanocrystals are known to have numerous advantages over organic dyes [13] as novel luminescent imaging probes for investigating biological systems. In fact, core-shell QDs of $\mathrm{CdSe} / \mathrm{ZnSe} / \mathrm{ZnS}$ have been designed for site-specific targeting of neurotransmitter uptake and transport. Examples include probes for sites of interaction and function of biogenic amine transporters for serotonin [14] and dopamine. [15] To target and investigate cholinergic sites, we reported the first example of water-soluble $\mathrm{CdSe} / \mathrm{ZnSe} / \mathrm{ZnS}$ QDs with an aminated analogue of hemicholium-15 (HC-15), a well-known inhibitor of CHT, attached to the surface of the QDs. [16] In this manuscript a new bioconjugate with a thiolated $\mathrm{Ch}$ analogue (Figure 1b) was synthesized and coupled to QDs to mimic the natural Ch substrate for CHT. The thiolated Ch derivative was designed with the important recognition elements for CHT, the quaternary ammonium and hydroxyl groups, and a terminal thiol to function as the tether to anchor the recognition label to the outer ZnS layer of the QDs. Together, QDs conjugated to $\mathrm{Ch}$ and a known inhibitor provide new chemical diagnostic probes for investigating choline transport.

The synthesis of ( $S$ )-2-hydroxy-3-mercapto- $N, N, N$-trimethylpropan-1-aminium chloride (Scheme 1) was developed from a method reported by Lewis and Sun for the synthesis of a mercaptoethyl quaternary ammonium derivative. [17] To produce $\mathrm{Ch}_{-} \mathrm{S}_{2} \mathrm{O}_{3}$, (S)-(-)-(3-chloro-2hydroxypropyl)-trimethylammonium chloride (Ch-Cl) $(11.3 \mathrm{~g}, 0.0601 \mathrm{~mol})$ and sodium thiosulfate $(14.9 \mathrm{~g}, 0.0942 \mathrm{~mol})$ were dissolved in deionized water $(160 \mathrm{~g}, 8.89 \mathrm{~mol})$. The $\mathrm{pH}$ was adjusted to 7.5 with $\mathrm{NaOH}$ and the mixture was refluxed for $7.5 \mathrm{~h}$. Water was removed under reduced pressure and the residue was stored under vacuum overnight. The solid product was rinsed twice with acetone and dissolved in as little water as possible. Acetone was added to precipitate the solid. The mixture was stirred and the precipitate was left to settle at the bottom. The supernatant was decanted off, and the solid was again dried under vacuum. A white solid was recovered and stored in a desiccator for further use.

The final product, $\mathrm{Ch}-\mathrm{SH}$, was prepared by sealing $\mathrm{Ch}_{-} \mathrm{S}_{2} \mathrm{O}_{3}(0.4 \mathrm{~g}, 1.39 \mathrm{mmol})$ in a microwave test tube purged with Ar. A solution of $\mathrm{HCl}(37 \%, 5.36 \mathrm{~g}, 14.7 \mathrm{mmol})$ in water ( $2.64 \mathrm{~g}, 146 \mathrm{mmol}$ ) was deoxygenated by an Ar purge and added to the sealed vial. The solution was heated by microwave irradiation for $30 \mathrm{~min}$ at $110^{\circ} \mathrm{C}$. After cooling, the solution was transferred to a round bottom flask, and the water was removed under reduced pressure. 
Anhydrous and deoxygenated ethanol was added to the dried powder, and allowed to stir for 30 min under Ar. The supernatant was collected and run through a pipette packed with cotton. The ethanol wash was repeated three times. The combined ethanol washings was either used immediately to make QD bioconjugates or concentrated to obtain a cloudy white honey-like substance that was stored under Ar for further use. Yields for both reaction steps were variable due to the presence of inorganic residues.

Ch-SH was successfully synthesized and the identity confirmed by NMR and mass spectrometry. CHN analyses however indicated purification was complicated by an inorganic byproduct in the reactions, most likely $\mathrm{NaCl}$. Not surprisingly, this resulted in variable yields for each reaction step. [17] Incorporation of additional rinsing steps with ethanol increased the purity of the product. Since the amount of $\mathrm{Ch}-\mathrm{SH}$ used for the preparation of the labeled QDs (Ch-S-QDs) was in excess, this procedure was sufficient to remove the majority of the excess salt. Remaining salt did not affect the formation of the Ch-S-QDs. It is also important to note the reaction time for conversion of $\mathrm{Ch}-\mathrm{S}_{2} \mathrm{O}_{3}$ to $\mathrm{Ch}-\mathrm{SH}$ was reduced from days to under an hour by heating the reaction mixture by microwave irradiation in a sealed tube under Ar. NMR spectroscopy also revealed complete conversion to $\mathrm{Ch}-\mathrm{SH}$ was achieved.

The choline-labeled QDs were prepared by first synthesizing the CdSe/ZnSe/ZnS core-shell QD [16] followed by ligand exchange of TOPO coated QDs with Ch-SH. Ch-SH was dissolved in ethanol and filtered through a cotton filled pipette. QDs $(0.1 \mathrm{~g})$ were added to the filtrate and this mixture was allowed to stir for $30 \mathrm{~min}$, followed by centrifugation for $2 \mathrm{~min}$. The ethanol containing unreacted ligand was finally decanted off. The precipitate was dissolved in $0.1 \mathrm{M}$ phosphate buffer (PBS) $\mathrm{pH} 7.3$ to which $\mathrm{CHCl}_{3}$ was added to extract residual organics from the aqueous solution. The exchange process was monitored by following the QD fluorescence under UV illumination in a 1:1 $\mathrm{PBS}: \mathrm{CHCl}_{3}$ mixture. Figure 2 depicts from left to right the fluorescence of the unmodified $\mathrm{CdSe} / \mathrm{ZnSe} / \mathrm{ZnS}$ QDs in the $\mathrm{CHCl}_{3}$ phase, the Ch-S-QDs in the aqueous phase after initial removal of impurities with $\mathrm{CHCl}_{3}$, and the water soluble Ch-S-QDs after cleaning with sufficient $\mathrm{CHCl}_{3}$ to completely remove all organic impurities. Figure 2 clearly demonstrates the formation of water soluble $\mathrm{Ch}-\mathrm{S}-\mathrm{QD}$ s by transfer to the aqueous phase following exchange of the organic capping groups with $\mathrm{Ch}-\mathrm{SH}$.

The Ch-S-QDs stored on the bench top in PBS or dried by rotary evaporation and stored in the dark in the vacuum oven maintained their solubility and fluorescence for over two months.

The ${ }^{1} \mathrm{H}$ NMR spectra for the Ch-SH ligand and the Ch-S-QDs are found in the supplementary materials in Figures S1-S3. Two distinct sets of peaks were observed indicating the presence of both free and bound forms of the Ch-SH ligand in the spectrum for the Ch-S-QDs (Figure S3). The peaks for $\mathrm{H}_{\mathrm{c}}$ at $4.34-4.21$ and $\mathrm{H}_{\mathrm{d}}$ at $2.71-2.60$ correspond to the free ligand in solution. The peaks moved downfield in the bound form with $\mathrm{H}_{\mathrm{c}}$ appearing at 4.57-4.44 ppm and $\mathrm{H}_{\mathrm{d}}$ at 2.97- $2.79 \mathrm{ppm}$. No such shift was observed for $\mathrm{H}_{\mathrm{a}}$ and $\mathrm{H}_{\mathrm{b}}$, which are further away from the surface of the QDs. The signal for $\mathrm{H}_{\mathrm{a}}$ was split into two peaks consistent with two ligand species present in solution. Peaks in the ${ }^{31} \mathrm{P}$ NMR spectra for the core-shell QDs were consistent with a TOPO and TOP coated surface. [16] Upon reaction with $\mathrm{Ch}-\mathrm{SH}$ the characteristic TOPO and TOP peaks disappeared providing further evidence that ligand exchange occurred. 
Figure 3 shows the comparison of the UV-visible spectra for the $\mathrm{CdSe} / \mathrm{ZnSe} / \mathrm{ZnS}$ core-shell QDs in $\mathrm{CHCl}_{3}$ to the Ch-S-QDs in 0.1 M PBS pH 7.3. A well-defined peak maximum centered at $564 \mathrm{~nm}$ is observed consistent with the formation of core-shell CdSe/ZnSe/ZnS QDs. Ligandexchange and water solubilization of the Ch-S-QDs yields a broad absorption for the first excitonic transition from 525 to $535 \mathrm{~nm}$, which was blue-shifted in comparison to the organic capped QDs.

Fluorescence spectra before and after ligand exchange with $\mathrm{Ch}-\mathrm{SH}$ are shown in Figure 4. A slight red shift in the wavelength of maximum fluorescence intensity was observed from the core-shell CdSe/ZnSe/ZnS QDs $(645 \mathrm{~nm})$ to the Ch-S-QDs $(663 \mathrm{~nm})$ due to the coordination of the thiol to the QD periphery. [18] Ligand exchange also led to a substantial decrease in fluorescence. The relative peak intensity fell from 6.8 to 0.2 . Previous literature reports indicate a reduction of the fluorescence intensity results upon thiol coordination $[16,19]$ and solubilization in water. [20] Some additional intensity may be regained through photobrightening although potentially accompanied by surface structural changes. [21]

A STEM image of the Ch-S-QDs is shown in Figure 5. The Ch-S-QDs exhibited diameters of approximately 5 to $6 \mathrm{~nm}$ with a range from 1.5 to $7.5 \mathrm{~nm}$. Close examination of the image reveals some elongation of particles resulting from asymmetric growth during the synthesis. As a result there exists some variation in particle morphology with the measured polydispersity of $21 \%$.

In summary, to the best of our knowledge we report the first choline-labeled CdSe/ZnSe/ZnS bioconjugate. The Ch-S-QDs are nanometer in size and exhibit the characteristic spectroscopic features in aqueous solution for $\mathrm{CdSe} / \mathrm{ZnSe} / \mathrm{ZnS}$ QDs. Their water solubility and high level of luminescence potentially make them a valuable tool to be used as a probe in cholinergic assays.

\section{ACKNOWLEDGMENT}

National Science Foundation Grant No. 0840474 supported the purchase of the SEM used in this research. Support from The University of Toledo is also gratefully acknowledged.

\section{REFERENCES}

[1] S.L. Murphy, J. Xu, K. Kochanek, Deaths: Preliminary Data for 2010. National Vital Statistics Reports [Online] 2012, 1-69.

[2] L.E. Hebert, P.A. Scherr, J.L. Bienias, D.A. Bennett, D.A. Evans, Arch. Neurol. 60 (2003) 1119-1122.

[3] N.C. Berchtold, C.W. Cotman, Neurobiol. Aging. 19 (1998) 173-189.

[4] J. Hardy, D. Allsop, Trends Pharmacol. Sci. 12 (1991) 383-388.

[5] G. Bartzokis, P.H. Lu, J. Mintz, J. Alzheimers Dis. 6 (2004) S53-59.

[6] G. Bartzokis, Neurobiol. Aging 32 (2011) 1341-1371.

[7] P.T. Francis, A.M. Palmer, M. Snape, G.K. Wilcock, J. Neurol. Neurosurg. Psychiatr. 66 (1999) 137-147.

[8] S.M. Ferguson, R.D. Blakely, Mol. Interventions 4 (2004) 22-37. 
[9] (a) P.R. Lockman, D.D. Allen, Drug Develop. Industr. Pharm. 28 (2002) 749-771; (b) F.M. Ribeiro, S.A.G. Black, V.F. Prado, R.J. Rylett, S.S.G. Ferguson, M.A.M. Prado, J. Neurochem. 97 (2006) 1-12; (c) T. Okuda, T. Haga, Y. Kanai, H. Endou, T. Ishihara, I. Katsura, Nat. Neuroscience 3 (2000) 122-125; (d) R.D. Blakely, L.J. DeFelice, A. Galli, Physiology 20 (2005) 225-231; (e) G. Rudnick, Neurotransmitter Transporters: Structure, Function and Regulation, Humana Press, Totowa, NJ, 1997, Ch. 3, pp 88-90.

[10] C. Oswald, S. H. J. Smits, M. Hönig, L. Sohn-Bösser, L. Dupont, D. Le Rudulier, L. Schmitt, E. Bremer J. Biol. Chem. 283 (2008) 32848-32859.

[11] (a) J. Lerner, Comp. Biochem. Physiol. 93 (1989) 1-9; (b) F. Batzold, R. Dehaven, M.J. Kuhar, N. Birdsall, Biochem. Pharmacol. 29 (1980) 2413- 2416; (c) R. Devés, R.M. Krupka, Biochim. Biophys. Acta 557 (1979) 469-485.

[12] (a) D.D. Wise, T.V. Barkhimer, P.A. Brault, J.R. Kirchhoff, W.S. Messer, Jr., R.A. Hudson, J. Chromatogr, B 775 (2002) 49-56; (b) T.V. Barkhimer, J.R. Kirchhoff, W.S. Messer, Jr., R.A. Hudson, Electrophoresis 23 (2002) 3699-3704; (c) T.V. Barkhimer, J.R. Kirchhoff, R.A. Hudson, W.S. Messer, Jr., Anal. Biochem. 339 (2005) 216-222; (d) S. Cai, J. Mukherjee, L.M.V. Tillekeratne, R.A. Hudson, J.R. Kirchhoff, Bioorg. Med. Chem. 15 (2007) 7042-7047; (e) T.V. Barkhimer, J. R. Kirchhoff, R.A. Hudson, W.S. Messer, Jr., L.M.V. Tillekeratne, Anal. Bioanal Chem. 392 (2008) 651-662.

[13] (a) X. Michalet, F.F. Pinaud, L.A. Bentolila, J.M. Tsay, S. Doose, G. Sundaresan, A.M. Wu, S.S. Gambir, S. Weiss, Science 307 (2005) 538-544; (b) A.H. Fu, W.W. Gu, C. Larabell, A.P. Alivisatos, Curr. Opin. Neurobiol. 15 (2005) 568-575.

[14] (a) O. Kovtun, I.D. Tomlinson, D.S. Sakrikar, J.C. Chang, R.D. Blakely, S.J. Rosenthal, ACS Chem. Neurosci. 2 (2011) 370-378; (b) H.A. Ki, P.K. Naoghare, B.K. Oh, J.W. Choi, J.M. Song, Anal. Biochem. 388 (2009) 23-27; (c) I.D. Tomlinson, M.R. Warnerment, J.N. Mason, M.J. Vergne, D.M. Hercules, R.D. Blakely, S.J. Rosenthal, Bioorg. Med. Chem. Lett. 17 (2007) 5656-5660; (d) I.D. Tomlinson, J.N. Mason, R.D. Blakely, S.J. Rosenthal, Bioorg. Med. Chem. Lett. 15 (2005) 5307-5310; (e) J.N. Mason, H. Farmer, I.D. Tomlinson, J.W. Schwartz, V. Savchenko, L.J. DeFelice, R.D. Blakely, S.J. Rosenthal, J. Neurosci. Methods 143 (2005) 3-25; (f) S. J. Rosenthal, A. Tomlinson, E.M. Adkins, S. bSchroeter, S. Adams, L. Swafford, J. McBride, Y. Wang, L.J. DeFelice, R.D. Blakely, J. Am. Chem. Soc. 124 (2002) 4586-4594.

[15] (a) Tomlinson, I.D.; Iwamoto, H.; Blakely, R.D.; Rosenthal, S.J. Bioorg. Med. Chem. Lett. 2011, 21, 1678-1682; (b) Tomlinson, I.D.; Mason, J.N.; Blakely, R.D.; Rosenthal, S.J. Bioorg. Med. Chem. Lett. 2006, 16, 4664-4667; (c) Tomlinson, I.D.; Mason, J.N.; Burton, J.N.; Blakely, R.D.; Rosenthal, S.J. Tetrahedron 2003, 59, 8035-8047.

[16] C. Gégout, M.L. McAtee, N.M. Bennett, L.M.V. Tillekeratne, J.R. Kirchhoff, Nanoscale 4 (2012) 4719-4725.

[17] D.M. Lewis, L.J. Sun, Color Tech. 119 (2003) 286-291.

[18] J. Aldana, Y.A. Wang, X. Peng, J. Am. Chem. Soc.123 (2001) 8844-8850.

[19] P. Reiss, J. Bleuse, A. Pron, Nano Letters 2 (2002) 781-784.

[20] C. Bullen, P. Mulvaney, Langmuir 22 (2006) 3007-3013.

[21] H. Asami, Y. Abe, T. Ohtsu, I. Kamiya, M. Hara J. Phys. Chem. B 107 (2003) 1256612568. 
a)<smiles>C[N+](C)(C)CCO</smiles>

b)<smiles>C[N+](C)(C)C[C@@H](O)CS</smiles>

Figure 1. Structure of a) choline and b) thiolated choline derivative (Ch-SH).

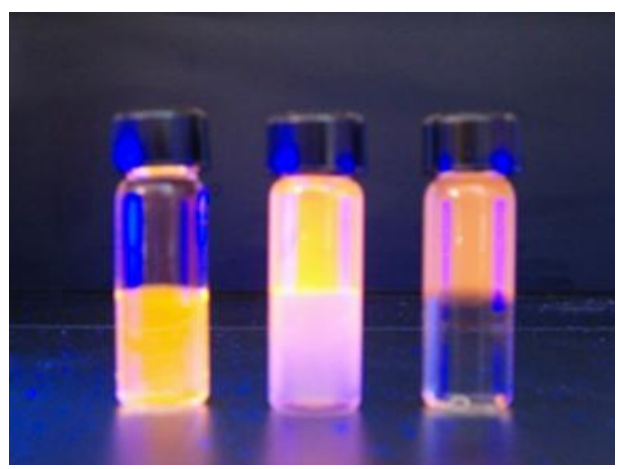

Figure 2. Left: CdSe/ZnSe/ZnS QDs, Center: Ch-S-QDs after initial cleaning with $\mathrm{CHCl}_{3}$, Right: $\mathrm{Ch}-\mathrm{S}-\mathrm{QDs}$ after thorough cleaning with $\mathrm{CHCl}_{3}$. Illumination by a UV lamp in 1:1 PBS: $\mathrm{CHCl}_{3}$.

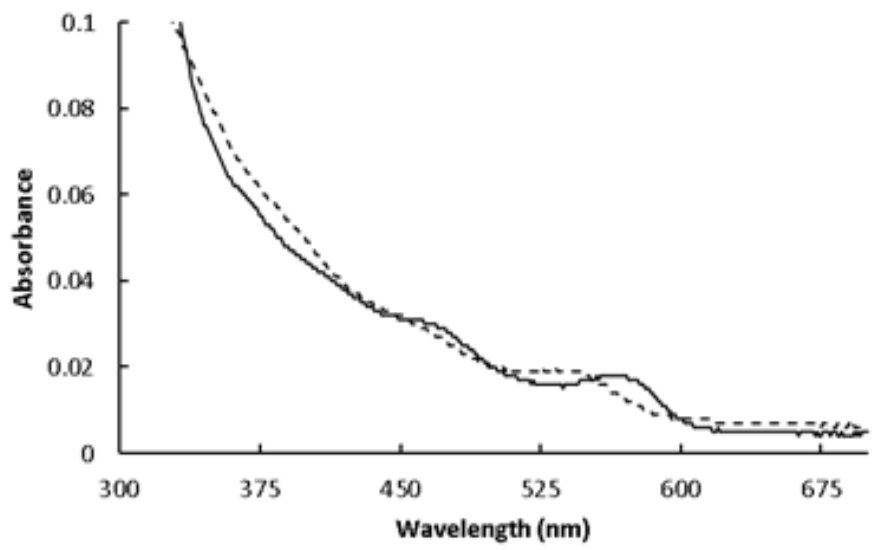

Figure 3. UV-visible spectra of (-) annealed $\mathrm{CdSe} / \mathrm{ZnSe} / \mathrm{ZnS}$ core-shell QDs in $\mathrm{CHCl}_{3}$ and (---) Ch-S-QDs in 0.1 M PBS pH 7.3. 


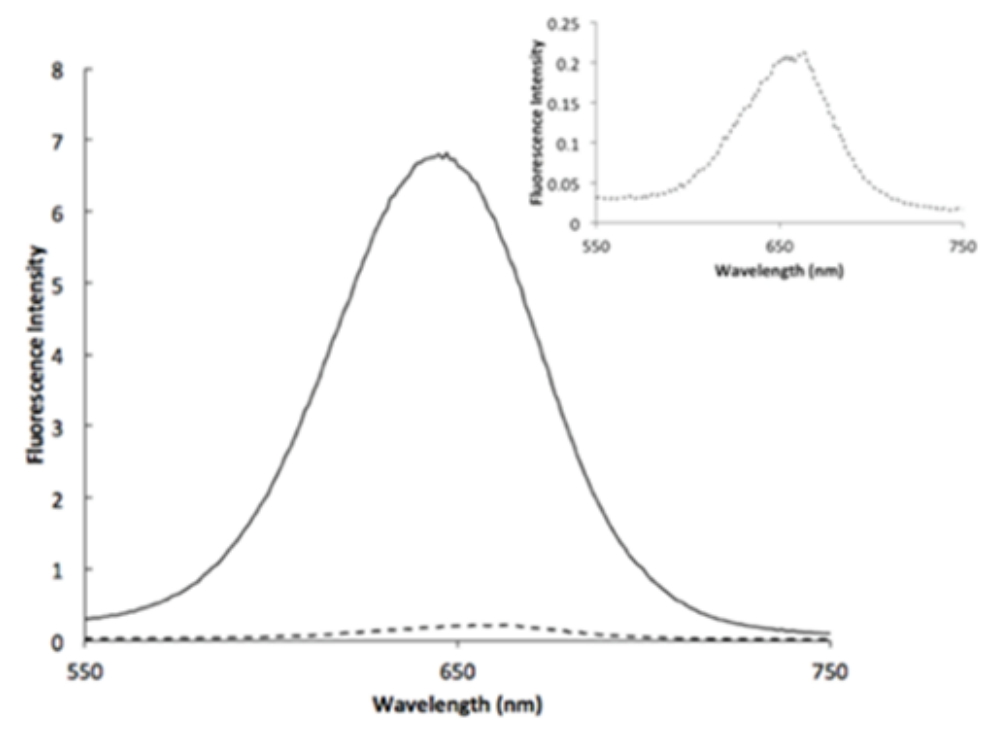

Figure 4. Fluorescence spectra of (-) annealed $\mathrm{CdSe} / \mathrm{ZnSe} / \mathrm{ZnS}$ core-shell QDs in $\mathrm{CHCl}_{3}$ and (---) Ch-S-QDs in 0.1 M PBS pH 7.3. Inset: Expanded version of the Ch-S-QD luminescence spectrum. The absorbance for each sample was matched at the excitation wavelength of $420 \mathrm{~nm}$.

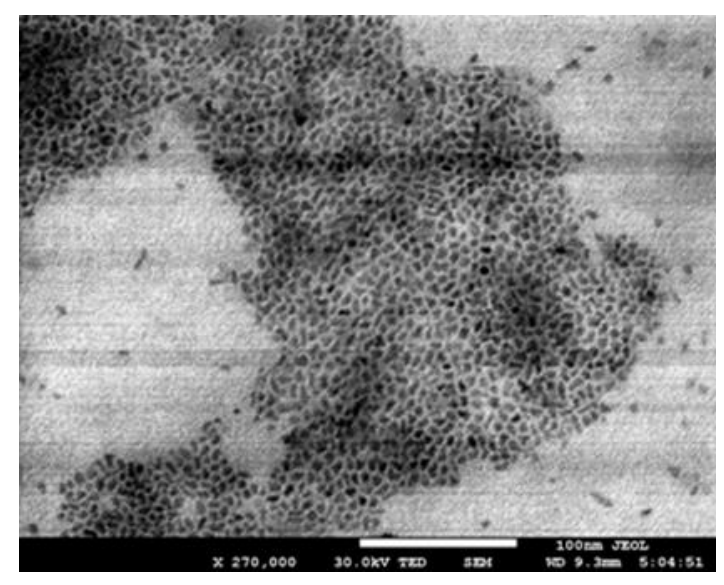

Figure 5. STEM image of Ch-S-QDs. 


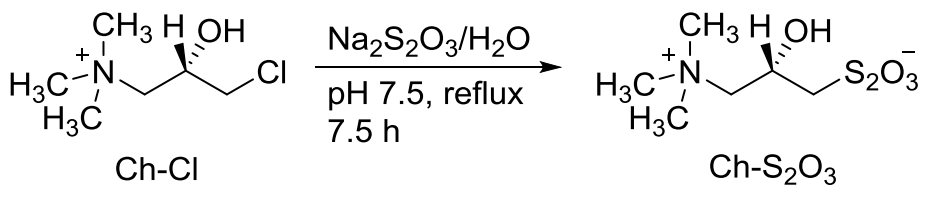

$$
\underset{\mu \mathrm{w}, 110^{\circ} \mathrm{C}}{\stackrel{\mathrm{HCl}(33 \% \mathrm{w} / \mathrm{w})}{\longrightarrow}} \mathrm{Ch}_{\mathrm{Ch}-\mathrm{SH}}
$$

Scheme 1. Synthesis of Ch-SH. 


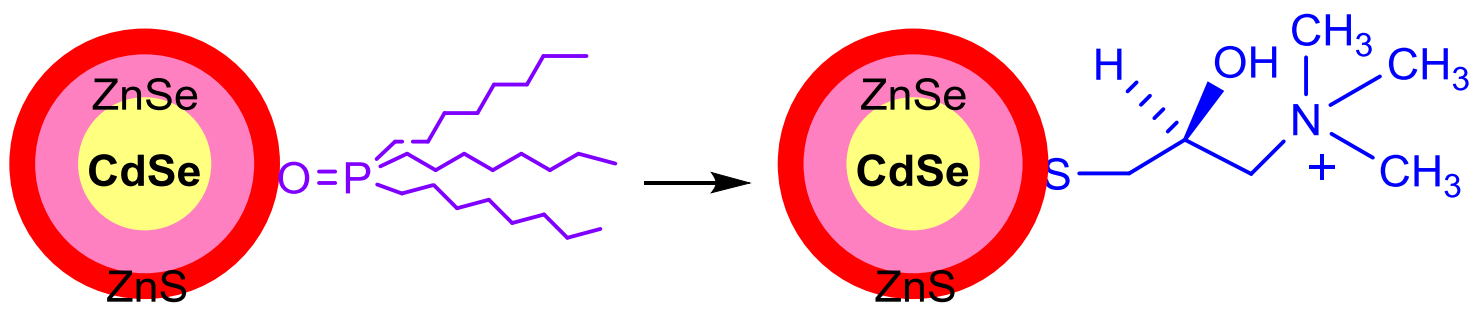
Core-shell
CdSe/ZnSe/ZnS water soluble cholinomimetic Ch-S-QDs

Graphical Abstract - Pictogram 


\section{Graphical Abstract - Synopsis}

Water soluble choline labeled CdSe/ZnSe/ZnS core-shell quantum dots were synthesized and exhibit strong luminescence in aqueous solution. 